\title{
Harmonic Analysis of Reactor and Capacitor in Single-tuned Harmonic Filter Application
}

\begin{abstract}
Jong-Gyeum Kim ${ }^{\dagger}$, Young-Jeen Park*, and Dong-Ju Lee**
Abstract - Industrial power distribution system includes many kinds of non-linear loads, which produce the harmonics during energy conversion transition. The single-tuned passive filter is widely used to absorb the harmonics and attenuate its undesirable effect in the distribution system. However, the passive filter might be severely stressed, and sometimes even damaged, due to the absorption of harmonics. There is voltage rise on the capacitor when the single-turned harmonic filter is applied. When the capacitor voltage rose above the allowable limit, the expected life of the capacitor will considerably deteriorate. On the other hand, the reactor can experience the spike voltage even if the voltage and current of the capacitor are within the allowable limit, and this accumulated voltage stress of the reactor causes its premature fault. In this paper, we analyzed and compared the harmonic voltage and current of the reactor and capacitor in a single-tuned harmonic filter through the EMTP software and verified them with the experimental results.
\end{abstract}

Keywords: Harmonics, Reactor, Capacitor, Resonance, Non-linear load

\section{Introduction}

In recent years, the modernization of industrial process and the sophistication of electrical equipment and machines have led to considerable development in power conversion applications.

Power conversion facilities such as uninterruptible power supply (UPS), rectifier, and adjustable speed drive (ASD) have been widely used in the power distribution system. These power conversion applications generate nonlinear current during the energy conversion process [1]-[5]. When these non-linear loads are used in the user's distribution system, voltage and current waveform are distorted. In addition, when the voltage and current harmonics are continued, the power quality of systems is deteriorated and becomes unsteady [4]-[6]. In order to obtain a stable and reliable system, there is a need for countermeasure such as passive or active filter.

Passive filters including the reactor and capacitor have long been used to mitigate special harmonics and are recently utilized at power factor compensation applications.

When series or parallel resonance exists in the circuit including the capacitor and reactor, the voltage magnification by parallel resonance has an effect on the insulation breakdown of the capacitor, and the high current by the series resonance may overheat the capacitors.

\footnotetext{
$\dagger$ Corresponding Author: Department of Electrical Engineering, Gangneung National University, Korea. (jgkim@gwnu.ac.kr)

* Department of Electrical Engineering, Gangneung National University, Korea. (popspark@gwnu.ac.kr)

** Department of Electrical Engineering, Chungnam Natioanl University, Korea. (1djn016@show.co.kr)

Received: May 11, 2010; Accepted: November 18, 2010
}

The experimental results of harmonic events showed that the fault rate of series reactor caused by harmonic is higher than that of power capacitor [2], [7]. The allowable limits of voltage and current for the capacitor were recommended, but accurate voltage and current information for reactor protection was not provided. Therefore, it is necessary to analyze the cause of reactor fault.

In this paper, we concluded that the voltage stress of reactor by harmonics is higher than that of capacitor, which can be major cause of fault.

\section{Capacitor and Reactor of Passive Harmonic Filter}

Loads like arc furnaces, rectifier and inverter units, battery charging equipment, thyristor power converters, motor control equipment, and static reactive power compensators are non-linear and responsible for introducing the harmonic currents into the distribution network and elsewhere [3], [4], [6]. Non-linear load generates a non-sinusoidal current from a sinusoidal source voltage. The non-sinusoidal current can be resolved into various harmonic components. The flow of harmonic currents in the line impedance produces harmonic voltages along with the fundamental frequency voltage at all points in the system.

A capacitor does not generate harmonics but easily absorb them. However, the reduced reactance of the capacitor to the higher frequencies magnifies the harmonic current in the circuit containing the capacitors. Harmonic voltage at the point at which the capacitor is connected will provide 
harmonic-order currents in the capacitor since capacitor impedance decreases along with increasing frequency, i.e., capacitor bank acts as a sink to all harmonics.

Capacitors are designed to withstand a reasonable amount of harmonics without detrimental effects. However, excessive harmonics at the capacitor bank leads to eventual failures of capacitor units [5]-[7].

When capacitors for power factor correction are applied to a system where harmonic exists, they can cause excessive harmonic voltage and current problems, leading to damage and breakdown.

Capacitors endure the greatest effect of voltage distortion [5], [7]. Even with a small amount of voltage distortion, they can overheat due to high RMS current. This is mainly caused by the capacitor's impedance characteristic, which is inversely proportional to the frequency. Capacitors will also suffer from insulation failure because of excessive peak voltages, which can be as high as the arithmetic sum of the harmonics.

The reactor is usually installed in series with a shuntconnected capacitor bank to limit inrush current due to switching and to control the resonant frequency of the system by the addition of the capacitor.

The capacitor-reactor combination acts as power compensation equipment at power frequency and as a filter at fifth harmonic. The reactor is serially connected to the capacitor to absorb the harmonic current and limit the inrush current in the user power application.

The capacitor and reactor are the major elements of a passive filter, which serve to increase the impedance of the capacitor against harmonic and shift the parallel resonance frequency of the source. In addition, the reactor absorbs a significant portion of the transient [8].

The reactor to the capacitor, which is designed to prevent system resonance, can be installed. It can also be designed to reduce the level of harmonics. With this application, a resonance phenomenon between network inductance and capacitor capacitance must be avoided.

Because passive filter is the most efficient and effective solution for harmonics problems, filter reactor is used in conjunction with capacitor bank to form a series-tuned harmonic filter circuit. This passive filter is generally installed in a shunt arrangement on the line side of the thyristor rectifier.

\section{Simulation and Measurement}

\subsection{Stress Analysis}

Fig. 1(a) shows a single-line diagram of an electrical distribution system feeding a thyristor rectifier for UPS application. Most power conversion devices are non-linear loads that tend to draw harmonics current at the line. If the line reactor is installed as in Fig.1(a), the harmonics components of the line current are somewhat reduced. Two line reactors, L1 and L2, are located at the front thyristor rectifier and act on current that are more sinusoidal. The passive

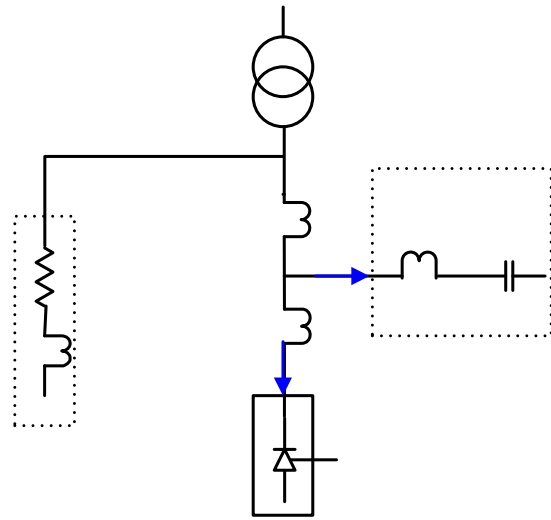

(a) Single-line diagram

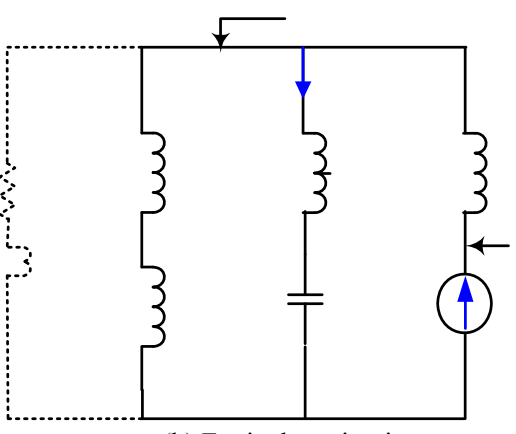

(b) Equivalent circuit

Fig. 1. Electrical distribution system feeding a thyristor rectifier.

filter is connected in parallel between the two reactors.

Fig. 1(b) illustrates the equivalent circuit of Fig. 1(a), which is used to calculate resonance frequency from harmonics sources.

Fig 2 shows the experimental set used in the study. All parameters for modeling and measurement of the system are shown in Table 1.

The harmonic current flowing into the capacitors causes excess heat in the capacitors, thereby reducing their lifetime. Thus, the reactor acts as a buffer.

The capacitor, which is connected to the reactor as part of a passive filter, will yield voltage that is above the nominal rated voltage. The voltage rise on the capacitor is expressed as follows [6]:

$$
\mathrm{V}_{\mathrm{c}}=\frac{\mathrm{V}}{1-\frac{\mathrm{P}}{100}}
$$

Where $\mathrm{p}$ is the relative impedance $\%$ value of the reactor for the capacitor at the filter. The reactor reactance is $\mathrm{p} \%$ of the capacitor reactance at the fundamental frequency. Connecting a $6 \%$ reactor in series with a capacitor produces a $6 \%$ rise in the $60 \mathrm{~Hz}$ voltage across the capacitor. Hence, the capacitor has to be rated for $106 \%$ of the normal system voltage. Otherwise, the normally available $10 \%$ over voltage capability of capacitors will be taken up by the $6 \%$ 


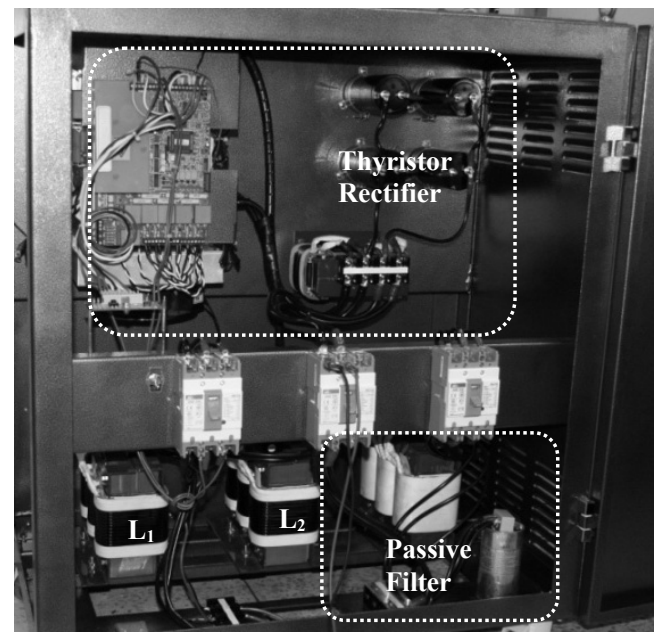

Fig. 2. Experimental set.

Table 1. System parameters

\begin{tabular}{c|c}
\hline Parameters & Value \\
\hline Power rating of input transformer & $30 \mathrm{kVA}$ \\
\hline Power rating of thyristor rectifier & $6 \mathrm{~kW}$ \\
\hline Inductance of input reactors $\left(\mathrm{L}_{1}, \mathrm{~L}_{2}\right)$ & $2.58 \mathrm{mH}$ \\
\hline Inductance of input transformer $\left(\mathrm{L}_{\mathrm{TR}}\right)$ & $0.7538 \mathrm{mH}$ \\
\hline Inductance of harmonic filter reactor $\left(\mathrm{L}_{\mathrm{f}}\right)$ & $2.85 \mathrm{mH}$ \\
\hline Capaciance of harmonic filter capacitor $\left(\mathrm{C}_{\mathrm{f}}\right)$ & $100 \mathrm{uF}$ \\
\hline
\end{tabular}

voltage rise, leaving only a $4 \%$ margin for system over voltage conditions.

Similarly, the voltage of the reactor is derived using (1). The reactor voltage, rather than capacitor voltage, is relatively low as shown in (2).

$$
\mathrm{V}_{\mathrm{L}}=\mathrm{V}_{\mathrm{C}} \times \frac{\mathrm{p}}{100}
$$

The capacitor will draw a large amount of harmonic component through the reactor in the passive filter. The reactor plays an important part in putting bounds to harmonic components.

The percentage of reactor is effective in the reduction of the reactance of capacitors by putting the reactors in series.

It is necessary to verify the possibility of the damage on the reactor being higher than that on the capacitor at the user power conversion system such as thyristor converter.

Fig. 3 shows the simulation result for the currents at both sides of the connected passive filter. The current $\left(\mathrm{L}^{2}\right)$ at the front side of the thyristor converter includes the harmonic component, whereas the current (L1) through the passive filter has considerably decreased harmonic components. The series-connected capacitor-reactor absorbs the harmonic current generated from the converter of the thyristor rectifier.

Fig. 4 shows the voltage and current of the reactor of the passive filter. The results of simulation and measurement are well fitted. The voltage across the reactor has two components,

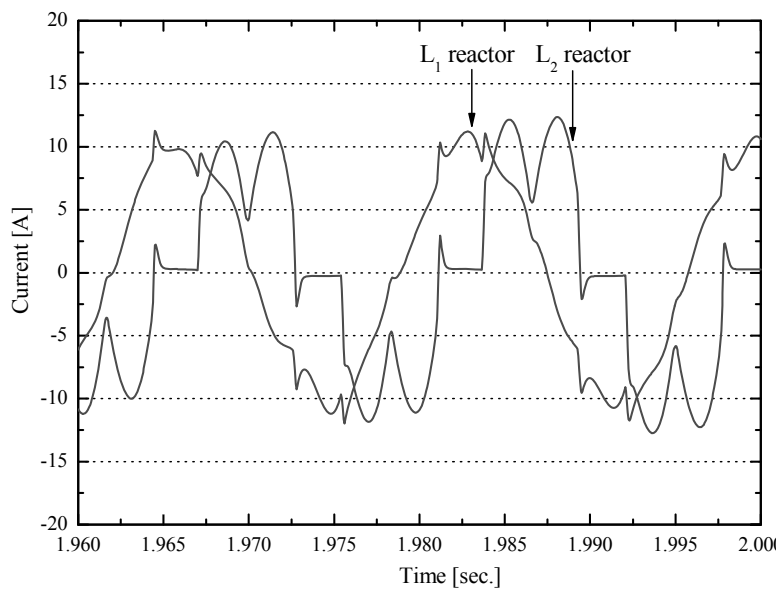

Fig. 3. $L_{1}$ and $L_{2}$ reactor current.

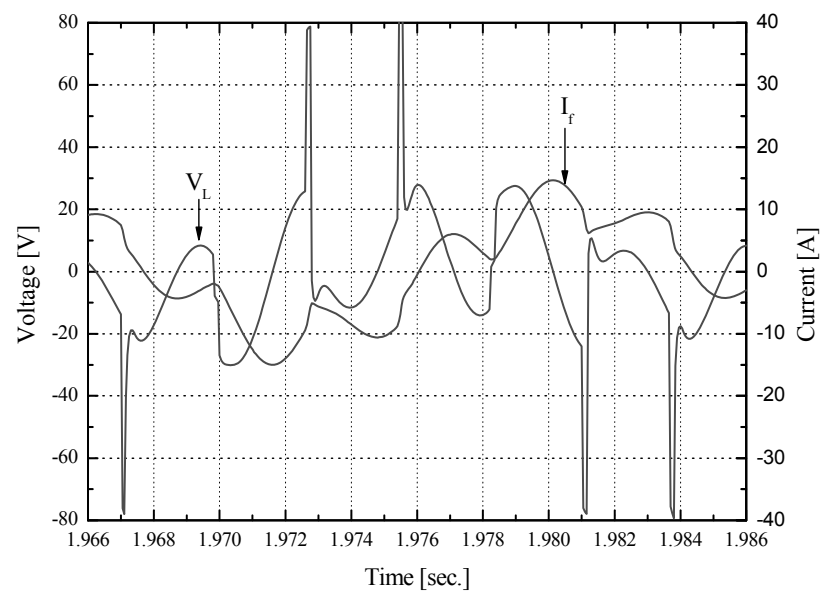

(a) Simulation results

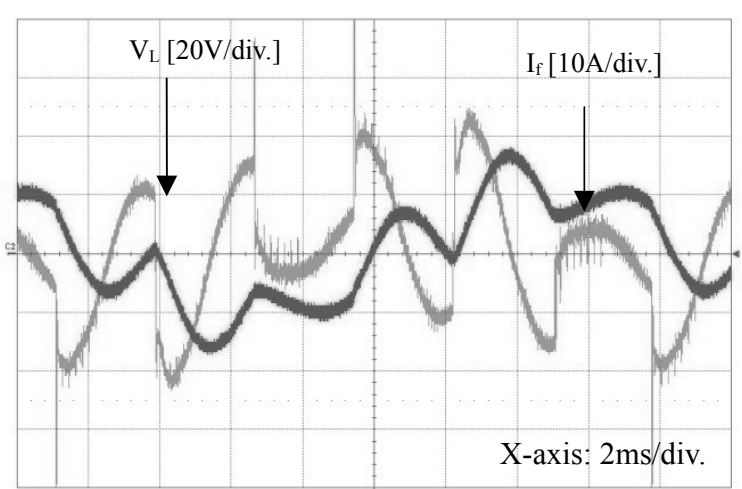

(b) Measurement result

Fig. 4. Voltage and current of reactor.

namely voltage drop and voltage peak. Aside from its rated voltage, the reactor will also show amplified harmonic voltage, which may overload the reactor bank and eventually lead to the failure of the reactor. From (2), the rated voltage of reactor is $22.8 \mathrm{~V}$, but the simulated RMS voltage is $25 \mathrm{~V}$ and the simulated peak voltage is $104 \mathrm{~V}$. Its peak value is 4.5 times the rated voltage. Therefore, the reactor can be under the instantaneous over voltage condition and can be saturated. 
Capacitors must be operated at or below their rated voltage. They can withstand continuous operation under contingency system and bank conditions provided that none of the following limitations is exceeded, as stated in Table 2 [1], [6]. There is no standard that addresses filter reactors, and most manufacturers use other guidelines such as that for transformers.

The Fourier spectrum of the reactor and capacitor voltage waveform from Fig. 4(a) are shown in Fig. 5. The largest

Table 2. Recommended practice for establishing capacitor capabilities

\begin{tabular}{c|c}
\hline RMS voltage across capacitor & $\leq 1.1 \times \mathrm{V}_{\mathrm{C}}$ \\
\hline Peak voltage limit across capacitor & $\leq 1.2 \times \sqrt{2} \times \mathrm{V}_{\mathrm{C}}$ \\
\hline Capacitor reactive power limit & $\leq 1.35 \times \mathrm{Q}_{\mathrm{C}}$ \\
\hline Capacitor RMS current & $\leq 1.35 \times \mathrm{I}_{\mathrm{C}}$ \\
& or $\leq 1.8 \times \mathrm{I}_{\mathrm{C}}$ \\
\hline
\end{tabular}

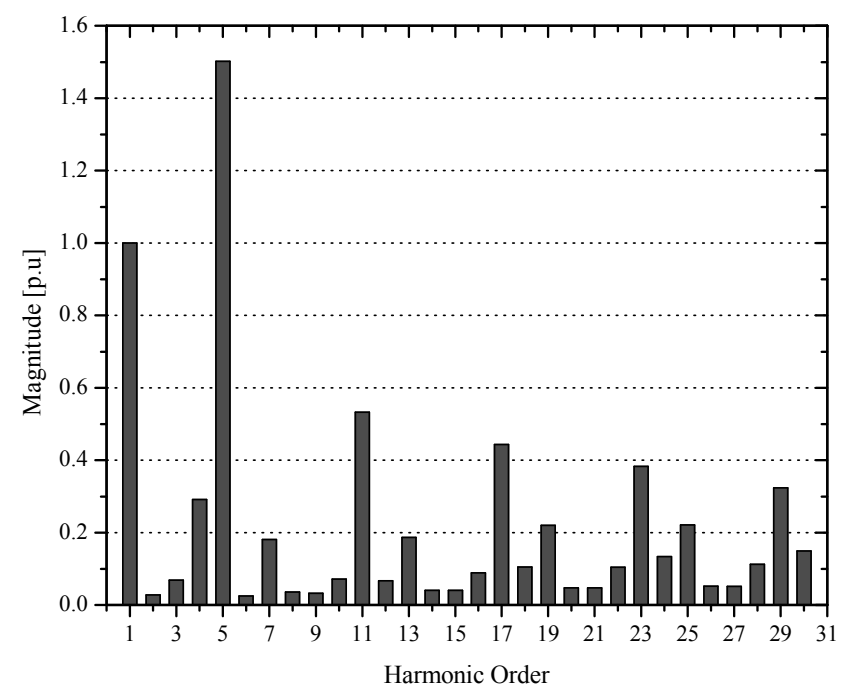

(a) Reactor voltage

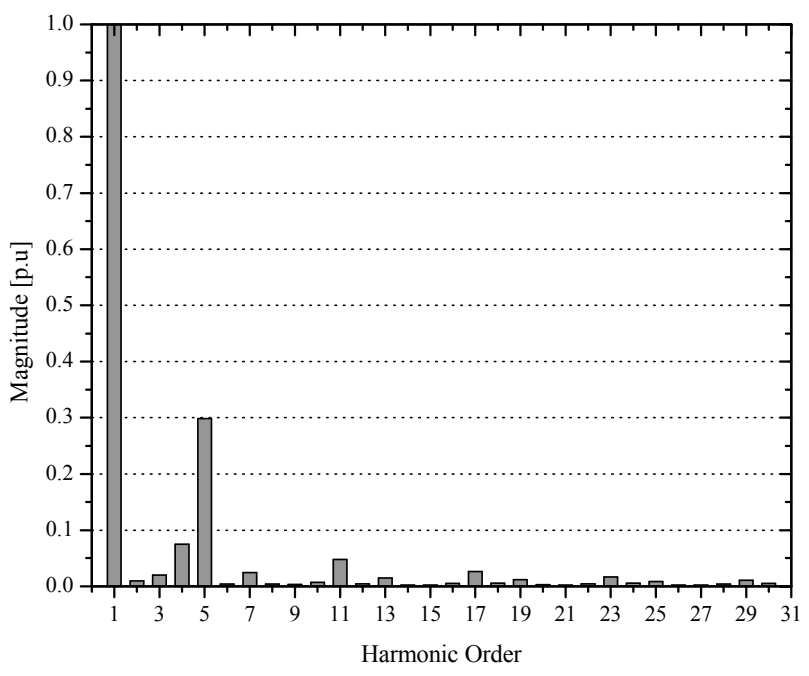

(b) Reactor current

Fig. 5. Fourier spectrum of rector voltage and current. existing harmonic order for the reactor is the fifth harmonic, which has a magnitude that is larger than the fundamental component and total voltage harmonic distortion (THD) amounting to $183.6 \%$. The harmonics of other orders are fairly included. Hence, the fifth passive filter can be damaged due to the harmonics. On the other hand, the voltage harmonic component of the capacitor is very low because of the series-connected reactor blocks off the harmonic components, with its THD being $6.56 \%$.

Fig. 6 shows the voltage and current of the capacitor of passive filter. The simulation waveform is similar to the measurement waveform. The voltage across the capacitor, rather than the reactor, is very sinusoidal. The current waveforms of the simulation and the measurement are equal to each other.

The Fourier spectrum for reactor voltage waveform from Fig. 6(a) is shown in Fig. 7. The harmonic order beside the fifth order component is very small and voltage stress barely acts on the capacitor.

From the analysis of Figs. 4 and 5, the reactor, rather than capacitor, is significantly influenced in the process of absorbing the harmonic components.

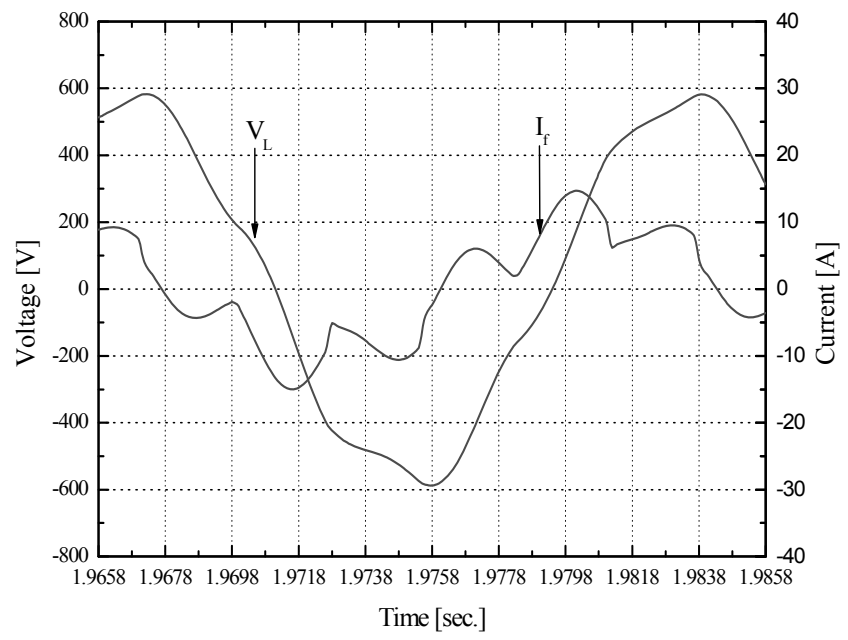

(a) Simulation

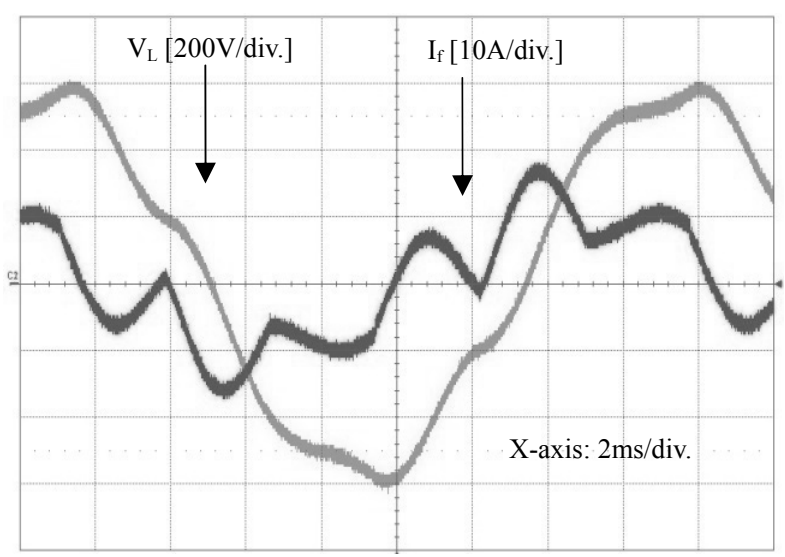

(b) Measurement

Fig. 6. Voltage and current of capacitor. 


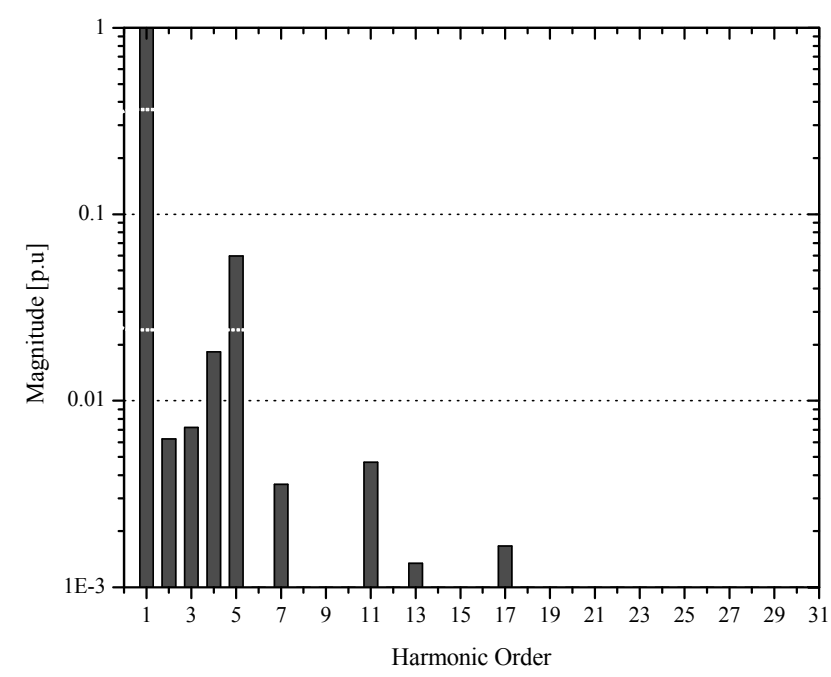

Fig. 7. Capacitor voltage harmonic spectrum.

\subsection{Analysis of Series and Parallel Resonances}

When a power system has a capacitor, there is a possibility that both a series and parallel resonance may be generated. Whereas series resonance results in extremely high harmonic currents, parallel resonance results in high voltages and currents, which can cause significant physical damage.

Impedance scans are performed without applications connected to the system for a worst-case condition analysis to determine where the resonant conditions exist. They are developed for both normal and abnormal operating conditions, as well as for future expansions. Impedance scans primarily refer to the impedance versus frequency plot of the system looking from the harmonic current source.

The impedance scan clearly shows the response of a system when capacitors are installed on the electrical system to form a part of a passive filter.

The impedance from the equivalent circuit of Fig. 1(b) with passive filter is given by (3) and (4). The denominator of the two equations has the same resonance frequency.

$$
\begin{gathered}
\mathrm{Z}_{1}(\mathrm{~s})=\frac{\mathrm{s}\left(\mathrm{L}_{1}+\mathrm{L}_{\mathrm{TR}}\right)\left(1+\mathrm{s}^{2} \mathrm{CL}_{\mathrm{f}}\right)+\mathrm{sL}_{2}+\mathrm{s}^{3} \mathrm{~L}_{2} \mathrm{C}\left(\mathrm{L}_{1}+\mathrm{L}_{\mathrm{f}}+\mathrm{L}_{\mathrm{TR}}\right)}{1+\mathrm{s}^{2} \mathrm{C}\left(\mathrm{L}_{1}+\mathrm{L}_{\mathrm{f}}+\mathrm{L}_{\mathrm{TR}}\right)} \\
\mathrm{Z}_{2}(\mathrm{~s})=\frac{\mathrm{s}\left(\mathrm{L}_{1}+\mathrm{L}_{\mathrm{TR}}\right)\left(1+\mathrm{s}^{2} \mathrm{CL}_{\mathrm{f}}\right)}{1+\mathrm{s}^{2} \mathrm{C}\left(\mathrm{L}_{1}+\mathrm{L}_{\mathrm{f}}+\mathrm{L}_{\mathrm{TR}}\right)}
\end{gathered}
$$

In Fig. 8, the impedance scan for resonance frequency at points 1 and 2 is shown. Point 1 is the reactor side through the thyristor converter, whereas point 2 is the connection point of the passive filter.

The frequency response for the two points to be simulated with electromagnetic transient program (EMTP)[10] is shown in Fig. 8. The two impedance scans with harmonic resonance near the fourth and fifth order harmonics are shown.

The frequency response for point 1 presents the possibil- ity of current amplification at the parallel resonance frequency of $203 \mathrm{~Hz}$ and voltage amplification at the series resonance frequency of $244 \mathrm{~Hz}$. The frequency for point 2 is calculated in the same way, with parallel and series resonance generated at 203 and $300 \mathrm{~Hz}$. The parallel impedance of point 2 is smaller than that of point 1, implying that passive filter effectively acts at point 2 .

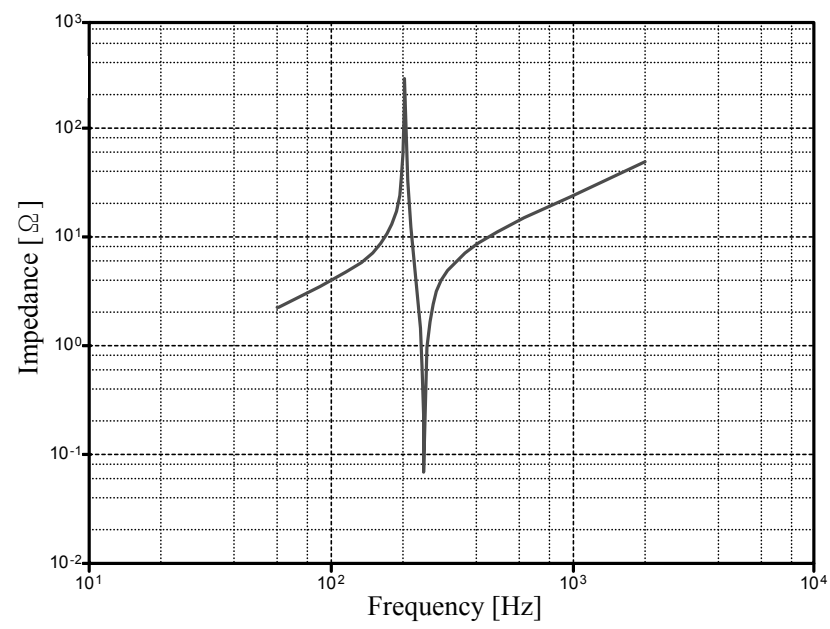

(a) At point 1

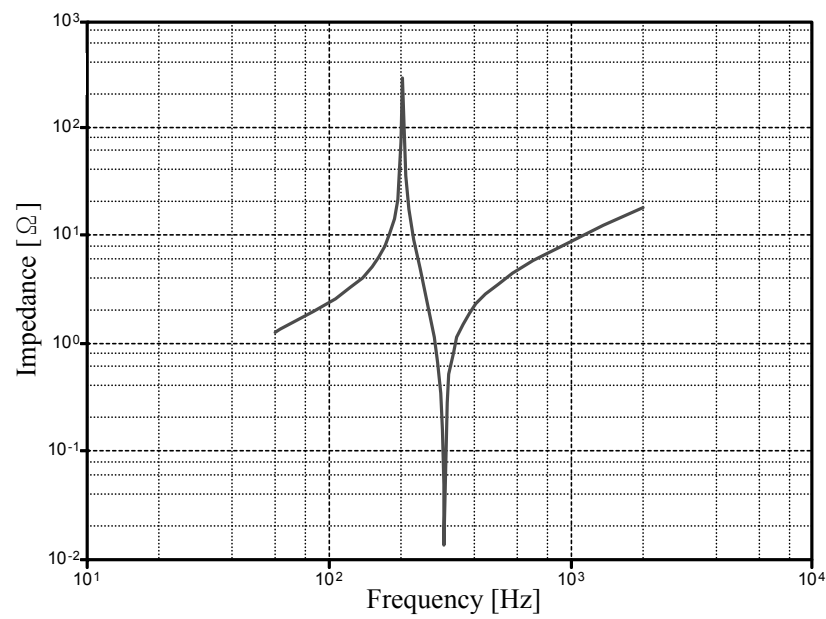

(b) At Point 2

Fig. 8. System response at points 1 and 2 .

\section{Conclusion}

This paper presents the electrical stress analysis of reactor and capacitor on passive filter. We found that the fifth harmonic voltage of reactor is clearly larger than fundamental voltage.

A reactor connected to capacitor in series was used to absorb the harmonic current being generated from the nonlinear load. This is significantly influenced by harmonic voltage and current. Reactor voltage was increased by the connection of capacitor, and its voltage was added by the harmonic component. 
It was verified by simulation and experimental results that voltage stress, such as spike voltage, during the transition of converter is an undesirable effect for the reactor, causing its premature fault.

\section{Acknowledgements}

This paper was recommended for outstanding paper award by the Intelec2009 Conference.

\section{References}

[1] IEEE Std.1531-2003, "Application and Specification of Harmonic Filters"

[2] Konishi Y. "Harmonic compensator-active filter", Proc of power quality symposium, pp.14-17, 1999. Sep.

[3] Douglas Andrews et al., "Harmonic Measurements, Analysis, and Power Factor Correction in a Modern Steel Manufacturing Facility", IEEE Trans on IAS, Vol. 32, No.3, pp.617-624, May/Jun. 1996

[4] IEEE Std 519-1992, "IEEE Recommended Practices and Requirements for Harmonic Control in Electrical Power Systems"

[5] Thomas M. Blooming, "Capacitor Application Issues", IEEE Trans on IAS, Vol. 44, No. 4, pp. 10131026, Jul/Aug. 2008.

[6] V. Suresh Kumar et al., "Harmonic Mitigation and Power Factor Improvement using Fuzzy Logic and Neutral Network Controlled Active Power Filter", Journal of Electrical Engineering \& Technology, Vol. 3, No. 4, pp.520-527, Dec 2008.

[7] Jong-Gyeum Kim et al., "Characteristics Analysis of Capacitor and Reactor for Harmonic Filter", KIEE, Vol. 58p. No. 1, pp.1-8, Mar., 2009.

[8] IEC 60076-6, "Power transformer-part 6: Reactors".

[9] BS EN 60829-1994, "Reactors".

[10] IEEE Standard for Shunt Power Capacitor, IEEE Std 18-2002.
[11] H. W. Dommel, "Electromagnetic Transients Program. Reference Manual (EMTP Theory Book)", BPA 1986.

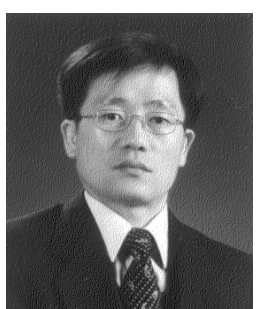

Jong-Gyeum Kim received his B.S. degree in Electrical Engineering from Dong-A University, Pusan, Korea in 1984 and M.S. and Ph.D. degrees in Electrical Engineering from Chungnam National University in 1991 and 1996, respectively. He is currently working for Gangneung-Wonju National University. His research interests include the design and implementation of energy conversion system and power quality.

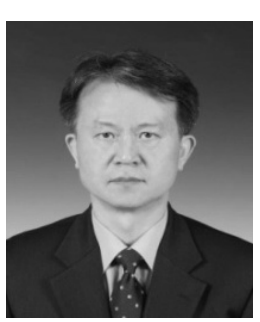

Young-Jeen Park received his B.S., M.S., and Ph.D. degrees in Electrical Engineering from Dankook University in 1982, 1986, and 1996, respectively. $\mathrm{He}$ is currently working for Gangneung-Wonju National University. His research interests include the design and application of automatic control

system.

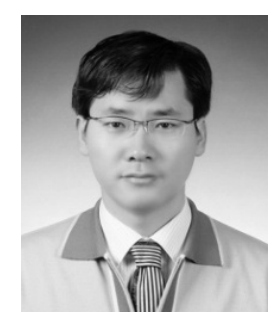

Dong-Ju Lee received his B.S. and M.S. degrees in Electrical Engineering from Chungnam National University in 1992 and 1998, respectively. He is currently working for Kukje Communication Co., Ltd. His research interests include the design and implementation of uninterruptible power supply system. 